\title{
Neuromuscular electrical stimulation exercise: a potential alternative to conventional exercise in the management of type 2 diabetes
}

\author{
OONAGH M GIGGINS, ${ }^{1,2}$ LOUIS CROWE, ${ }^{1}$ GARRETT F COUGHLAN, ${ }^{1}$ BRIAN CAULFIELD ${ }^{1,2}$
}

\begin{abstract}
Aims: Exercise is fundamental in the prevention and treatment of type 2 diabetes (T2D). However, many individuals face barriers to exercise. Neuromuscular electrical stimulation (NMES) is an alternative to conventional exercise that may prove beneficial in the treatment of T2D. The aim of this study was to investigate the effects of an 8-week NMES exercise programme in a T2D population.

Methods: A repeated measures one-group cohort intervention study was conducted. Thirteen T2D participants (age 52.0 \pm 6.9 years, height $1.79 \pm 0.06 \mathrm{~m}$, weight $104.5 \pm 11.9 \mathrm{~kg}$, BMl $32.8 \pm 4.3$ $\mathrm{kg} / \mathrm{m}^{2}$ ) underwent an 8-week NMES intervention. Venous blood markers, body composition, blood pressure, quadriceps strength and predicted maximal oxygen consumption were assessed at baseline and after the 8-week intervention.

Results: Significant improvements in fasting plasma glucose, percentage body fat and peak isometric quadriceps torque were noted following the intervention $(p<0.05)$.

Conclusions: The principal findings of this study were that NMES can improve body composition, muscle strength and glycaemic control in T2D participants. NMES may therefore provide an alternative to those individuals with T2D who have barriers to exercise participation. Further randomised controlled trials with larger participant numbers are required to investigate this further.
\end{abstract}

Br J Diabetes 2017;17:46-51

Key words: neuromuscular electrical stimulation, exercise, type 2 diabetes, glycaemic control

School of Public Health, Physiotherapy and Sports Science, Health Science Centre, UCD, Befield, Dublin, Ireland

Insight Centre for Data Analytics, O'Brien Centre for Science, UCD, Befield, Dublin, Ireland

Address for correspondence: Dr Oonagh M Giggins School of Public Health, Physiotherapy and Sports Science, Health Science Centre, UCD, Befield, Dublin, Ireland

E-mail: oonagh.giggins@ucd.ie

http://dx.doi.org/10.15277/bjd.2017.127

\section{Introduction}

The number of people with type 2 diabetes (T2D) is increasing due to population growth, coupled with an increasing prevalence of physical inactivity and obesity. Exercise is vital in the management of T2D. ${ }^{1-3}$ However, despite the benefits of engaging in physical activity, many individuals with T2D still lead a sedentary lifestyle, with only $34 \%$ of individuals taking some form of physical activity within a random two-week window. ${ }^{4}$ Many individuals face barriers to exercise participation secondary to complications of their condition (eg, peripheral vascular disease, neuropathy) or other musculoskeletal and orthopaedic problems (eg, osteoarthritis). In addition, many face psychological barriers to exercise; lack of self-efficacy, feelings of tiredness, distraction by good television programmes, lack of time, fear of diabetes getting worse, poor weather and feeling depressed have all been reported as barriers to exercise in individuals with T2D. ${ }^{4}$ It is clear that current exercise interventions are unsuitable for these individuals, and an alternative form of exercise is required.

Neuromuscular electrical stimulation (NMES) is the application of electrical stimulation to the peripheral nervous system provoking a muscle contraction. NMES has received much attention in recent years as a means of inducing exercise, resulting in significant improvements in maximal aerobic capacity, muscle strength and capacity for physical activity in both sedentary adults $^{5}$ and patients with chronic heart failure. ${ }^{6}$ More recently, this form of NMES exercise was found to induce a cardiovascular response and to significantly increase energy expenditure in an obese population. ${ }^{7}$

Evidence exists to suggest that NMES may be used as an alternative to conventional exercise in T2D. Early studies in healthy individuals showed that whole body glucose uptake could be substantially enhanced during and after a single bout of NMES.8,9 Subsequently it was shown that postprandial glucose levels could be significantly attenuated by 30 min of NMES applied to the lower limbs of patients with T2D. ${ }^{10} \mathrm{~A}$ more recent study also showed that NMES could enhance glucose metabolism in patients with T2D. ${ }^{11}$ The effects of NMES on insulin sensitivity has also been examined in T2D patients, with the results of this study demonstrating that insulin sensitivity can be 
improved after a single session of NMES and after a week of daily training. ${ }^{12}$ The effects of prolonged daily NMES training has also been investigated in individuals with T2D. ${ }^{13}$ The findings of this study indicated that, while NMES can acutely increase energy expenditure, glucose uptake and cardiovascular responses in T2D, no clinical benefits were observed following 12 weeks of training.

Perceived discomfort limits the effectiveness of prolonged NMES training. We have therefore modified the traditional approach to NMES in an effort to improve comfort levels. By increasing electrode size, the current density at the skin surface can be reduced, thereby reducing discomfort while maintaining the efficacy of the electrical stimulation. ${ }^{14}$ Our stimulation also differs from traditional NMES in that it delivers trains of pulses in unconventional pathways. Larger electrodes arranged in arrays rather than pairs permit multiple pathways for the current to flow. This form of stimulation allows greater stimulating currents to be tolerated, thus producing stronger, more comfortable muscle contractions. Therefore, the objective of this study was to investigate whether 8 weeks of training with our NMES stimulation can have a beneficial effect on aerobic fitness, muscle strength, body composition, heart rate, blood pressure as well as the blood markers of diabetes in a population of individuals with T2D.

\section{Methods}

\section{Subjects}

Thirty-nine male participants were recruited from a diabetes clinic in a local university hospital and from an advertisement in a national newspaper. Inclusion criteria were patients with T2D aged between 18 and 65 years. Only male participants were included as previous pilot testing showed that female participants with increased body fat did not tolerate the NMES parameters used in this investigation. Other exclusion criteria included the presence of any cardiovascular conditions or any other conditions which are contraindicated to NMES. Medications were not altered during the course of the study. Potential participants were assessed for their eligibility to take part in the study over the telephone. Eligible candidates gained clearance from their general practitioner/endocrinologist prior to their participation in the study. Upon the request of their doctor, participants underwent an exercise stress test.

This study was approved by the local university hospital and university research ethics committees and written informed consent was obtained from all study participants.

\section{Design}

This study was a repeated measures single group cohort intervention study.

\section{Measurements}

Experimental measures were assessed at baseline and after the intervention period. Participants arrived at the laboratory in the morning following an overnight fast, abstaining from their usual glucose-lowering medical treatment. They were instructed to avoid strenuous physical activity on the day before testing. At baseline, participants completed a food diary for the day before and were then asked to replicate their food intake the day before the follow-up testing session. The following measurements were conducted at baseline and follow-up.

\section{Venous blood markers}

A fasting blood sample was obtained to determine the blood markers of diabetes control: fasting plasma glucose and $\mathrm{HbA}_{1 c}$. The blood sample was also analysed to determine the blood lipid profile: total cholesterol, high-density lipoprotein cholesterol, low-density lipoprotein cholesterol and triglycerides.

\section{Body composition}

A DEXA scan (Lunar, GE Healthcare, Madison, WI, USA) was performed to determine total body mass, percentage body fat, lean body mass and android/gynoid ratio.

\section{Blood pressure and heart rate}

Blood pressure and resting heart rate were measured using a digital automatic blood pressure monitor (Omron Healthcare Europe BV, The Netherlands) with the participant in a seated position.

\section{Isometric strength test}

Dynamometry testing of the right quadriceps femoris was conducted on a Cybex dynamometer (Cybex Humac ${ }^{\circledR} / \mathrm{NORM}^{\mathrm{TM}}$, Model 770, Computer Sports Medicine, Stoughton, MA, USA). Participants were comfortably seated on the dynamometer chair with the hip joint at $90^{\circ}$. The dynamometer lever arm was attached $2-3 \mathrm{~cm}$ above the lateral malleolus using a strap. The chair was placed in a position so that the lateral femoral condyle was aligned with the axis of rotation of the dynamometer. The placement of the chair relative to the dynamometer for each individual was recorded to establish a standardised body position for test reproducibility. The upper leg, contralateral limb and shoulders were stabilised during testing and participants positioned their arms across their chest for the test. The testing protocol involved one set of four repetitions of isometric quadriceps contractions at $60^{\circ}$. Prior to the performance of the strength test, each participant performed a 3-min warm-up on a cycle ergometer (Lode BV, Medical Technology, The Netherlands). Each participant was given a practice trial of the test to familiarise themselves with the testing protocol. Visual feedback and standardised verbal encouragement were provided throughout the test.

\section{Predicted maximal aerobic capacity}

A submaximal aerobic exercise capacity test was performed using an incremental cycle ergometer test protocol ${ }^{15}$ with simultaneous cardiopulmonary gas exchange analysis (Quark b2, Cosmed, Italy) and heart rate measurement (Polar Electro, Tampere, Finland). Participants cycled on the ergometer as per protocol until they achieved $85 \%$ of their maximum heart rate, at which point the test was stopped. Heart rate was plotted against oxygen consumption and the graph was extrapolated to calculate the predicted maximal oxygen consumption $\left(\mathrm{VO}_{2} \mathrm{max}\right)$.

\section{NMES protocol}

A battery-powered low frequency muscle stimulator (NT2010, BioMedical Research, Galway, Ireland) was used to elicit rapid rhythmical contractions in the large lower extremity muscle groups. Impulses were delivered through four electrodes $(17 \mathrm{~cm}$ 
Figure 1. Location of stimulating electrodes
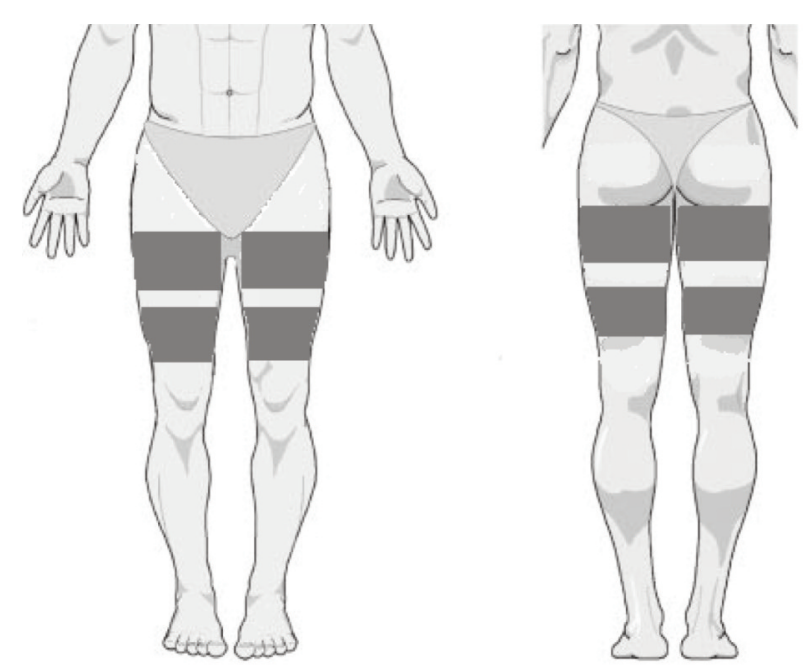

Table 1 Stimulation parameters

\begin{tabular}{llll}
\hline Phase & $\begin{array}{l}\text { Duration } \\
(\mathbf{m i n})\end{array}$ & $\begin{array}{l}\text { Frequency } \\
\mathbf{( H z )}\end{array}$ & Purpose \\
\hline 1 & 5 & 4 & $\begin{array}{l}\text { Warm-up; non-tetanic } \\
\text { contractions } \\
\text { Aerobic training; non-tetanic } \\
\text { contractions }\end{array}$ \\
\hline 3 & 40 & 5 & $\begin{array}{l}\text { Gradual increase in stimulation } \\
\text { current }\end{array}$ \\
4 & 5 & 19 & $\begin{array}{l}\text { Strength training; tetanic } \\
\text { contractions; } 5 \text { s contraction- } \\
\text { relaxation cycle }\end{array}$ \\
\hline 5 & 4 & 19 & $\begin{array}{l}\text { Strength training; continuous } \\
\text { tetanic contractions } \\
\text { Strength training; continuous } \\
\text { tetanic contractions } \\
\text { supplemented with voluntary } \\
\text { contractions } \\
\text { Cool down; non-tetanic } \\
\text { contractions }\end{array}$ \\
\hline 7 & 3 & 19 & \\
\hline
\end{tabular}

$\times 10.3 \mathrm{~cm}$ ) placed on the anterior and posterior aspect of each thigh (Figure 1). The electrodes were applied to the body via custom-made neoprene wrap garments fastened to the thigh with Velcro straps. The maximum peak output pulse current was 200 milliamps $(m A)$. The stimulation programme consisted of seven phases (phases 1-7) with parameters designed to produce both aerobic and strength training effects (Table 1). The pulse width for each phase was $760 \mu$ s and participants were instructed to adjust the stimulation intensity to the maximum tolerable level during the 1-hour session. During phase 6, participants were instructed to supplement the stimulation with voluntary contractions by extending and flexing their knee joint. Participants were requested to complete six 1-hour training sessions per week
Table 2 Venous blood markers, body composition, blood pressure, resting heart rate, peak isometric quadriceps femoris torque and predicted $\mathrm{VO}_{2}$ max at baseline and follow-up

\begin{tabular}{|c|c|c|c|}
\hline & Baseline & Follow-up & $p$ value \\
\hline Fasting plasma glucose $(\mathrm{mmol} / \mathrm{L})$ & $9.2 \pm 3.0$ & $7.8 \pm 2.4$ & $0.003^{*}$ \\
\hline \multirow[t]{2}{*}{$\mathrm{HbA}_{1 \mathrm{c}}(\%) \mathrm{mmol} / \mathrm{mol}$} & $8.0 \pm 1.6$ & $7.7 \pm 1.7$ & 0.221 \\
\hline & $64 \pm 17.5$ & $61 \pm 18.6$ & \\
\hline Triglycerides (mmol/L) & $1.8 \pm 0.9$ & $1.7 \pm 0.6$ & 0.722 \\
\hline $\begin{array}{l}\text { High-density lipoprotein cholesterol } \\
(\mathrm{mmol} / \mathrm{L})\end{array}$ & $1.1 \pm 0.2$ & $1.1 \pm 0.2$ & 0.929 \\
\hline $\begin{array}{l}\text { Low-density lipoprotein cholesterol } \\
(\mathrm{mmol} / \mathrm{L})\end{array}$ & $2.4 \pm 0.9$ & $2.3 \pm 0.7$ & 0.592 \\
\hline Total cholesterol (mmol/L) & $4.2 \pm 0.8$ & $4.0 \pm 0.7$ & 0.533 \\
\hline Android/gynoid ratio & 1.41 & 1.44 & 0.153 \\
\hline Lean body mass (kg) & $65.2 \pm 7.3$ & $65.3 \pm 7.1$ & 1.00 \\
\hline Total mass (kg) & $101.9 \pm 12.6$ & $100.6 \pm 11.9$ & $0.05^{\star}$ \\
\hline Percentage body fat & $33.6 \pm 4.3$ & $32.6 \pm 4.6$ & $0.01 *$ \\
\hline Systolic blood pressure (mm Hg) & $127 \pm 9$ & $123 \pm 7$ & 0.195 \\
\hline Diastolic blood pressure $(\mathrm{mm} \mathrm{Hg})$ & $87 \pm 8$ & $83 \pm 10$ & $0.032^{*}$ \\
\hline Resting heart rate (bpm) & $68 \pm 8$ & $66 \pm 7$ & 0.422 \\
\hline Isometric quadriceps torque (nm) & $173.5 \pm 60.5$ & $197.1 \pm 55.2$ & $0.016^{*}$ \\
\hline Predicted $\mathrm{VO}_{2} \max (\mathrm{ml} / \mathrm{kg} / \mathrm{min})$ & $31.4 \pm 4.4$ & $32.3 \pm 4.4$ & 0.552 \\
\hline $\begin{array}{l}\text { Data presented as mean } \pm \mathrm{SD} \text {. } \\
{ }^{*} \text { Indicates a statistically significant finding }\end{array}$ & & & \\
\hline
\end{tabular}

after their evening meal for 8 weeks. Every 15 min during training, participants recorded the maximum stimulation intensity achieved. A weekly telephone call or email was made to all participants to ensure adherence to the programme. Participants were not required to make any dietary/lifestyle changes, nor were they requested to maintain a food diary or physical activity log during the intervention period.

\section{Statistical analysis}

The statistical software package SPSS 18.0 for Windows (SPSS, Chicago, IL, USA) was used to analyse the data collected. All data were expressed as mean \pm standard error. The non-parametric Wilcoxon signed rank test was used to test for differences within the group at baseline and follow-up. An $\alpha$ level of $p \leq 0.05$ was set for all analyses.

\section{Results}

Thirty-nine men with T2D were recruited. Following screening, 16 participants were excluded as they had a cardiovascular condition and 10 participants withdrew/dropped out during the intervention period. Therefore, 13 T2D participants (age 52.0 \pm 6.9 years, height $1.79 \pm 0.06 \mathrm{~m}$, weight $104.5 \pm 11.9 \mathrm{~kg}$, body mass index $32.8 \pm 4.3 \mathrm{~kg} / \mathrm{m}^{2}$ ) completed the study. Seven participants 
were taking ACE inhibitors, eight lipid-lowering therapy, five aspirin and 12 were taking oral hypoglycaemic drugs. Participants reported no dietary, medication or activity changes during the intervention period.

Participants completed a minimum of 40 NMES sessions during the intervention period, training at stimulation intensities of 80-170 mA. Table 2 summarises all measurements obtained at baseline and follow-up. Fasting plasma glucose reduced significantly $(p<0.05)$ from baseline to follow-up. No other significant changes were observed in blood markers from baseline to followup. Significant reductions in total body weight and percentage body fat were observed following the 8-week intervention period. Non-significant changes were observed in other body composition measurements. A statistically significant reduction from baseline was measured for diastolic blood pressure at the 8-week followup. A significant increase in peak isometric quadriceps femoris torque was measured following the NMES intervention, however the observed increase in predicted $\mathrm{VO}_{2}$ max following the intervention was not statistically significant.

\section{Discussion}

This study showed that an 8-week NMES exercise programme improved body composition, muscle strength and glycaemic control in T2D participants. Exercise is a vital component in the management and prevention of T2D, but many individuals with T2D face barriers to exercise participation. NMES has been suggested as an alternative to conventional exercise; however, prior to this study, there has been a lack of evidence showing the beneficial effects of a prolonged NMES exercise intervention programme in a T2D population.

Clinically significant reductions in fasting plasma glucose were noted following the 8-week intervention. The current goal for fasting plasma glucose is 3.9-7.2 $\mathrm{mmol} / \mathrm{L}$. ${ }^{2}$ Following the intervention, the mean fasting plasma glucose level was closer to this target than at baseline. Lowering fasting plasma glucose levels is strongly associated with reductions in the risk of complications such as retinopathy, nephropathy and neuropathy. 16 However, the change in fasting plasma glucose levels after the NMES intervention are modest and fasting plasma glucose levels correlate poorly with $\mathrm{HbA}_{1 c}$, which is considered the optimal method of measuring long-term glycaemic control. A small nonsignificant decrease $(0.3 \%)$ was measured in $\mathrm{HbA}_{1 c}$ following the NMES intervention, which is unlike a previous study by the authors which demonstrated a significant improvement in $\mathrm{HbA}_{1 \mathrm{c}}$ levels following an NMES intervention. ${ }^{17}$ The dissimilar results obtained may be explained by the difference in stimulation parameters employed in the two studies. While the observed improvement in $\mathrm{HbA}_{1 \mathrm{c}}$ was not statistically significant, the change may be clinically meaningful. Church and colleagues reported a $0.3 \%$ reduction in $\mathrm{HbA}_{1 \mathrm{c}}$ levels following a 9-month combined aerobic and resistance training intervention, concluding that this may produce a $5-7 \%$ reduction in the risk of cardiovascular disease and a 12\% reduction in the risk of microvascular complications. ${ }^{18}$ Therefore, while the reduction observed in $\mathrm{HbA}_{1} \mathrm{c}$ in this present study was small, it may help reduce the risk of secondary complications of T2D.
A modest yet statistically significant reduction in percentage body fat was noted following the NMES training, but no significant increase was noted in lean body mass. It is likely that the aerobic training component of the NMES exercise resulted in greater calorie consumption and therefore yielded greater reductions in percentage body fat. Decreased fat mass results in increased insulin sensitivity ${ }^{19}$ and consequently improved glycaemic control. Therefore, by improving body composition, NMES may be applied to assist glycaemic control in T2D. Increased fat oxidation will also result in lower plasma non-esterified free fatty acid (NEFA) levels in obese T2D patients, and lowering plasma NEFA can reduce insulin resistance/hyperinsulinaemia and improve glucose tolerance in obese patients with $T 2 \mathrm{D} .{ }^{20} \mathrm{It}$ is therefore possible that the reduced plasma NEFA levels resulted in the improvements in fasting blood glucose by NMES. However, as plasma NEFA levels were not measured in this current investigation, this conclusion cannot be drawn.

The present investigation suggests that aerobic fitness can be improved in people with T2D through 8 weeks of NMES exercise, increasing by $3 \%$ from baseline to follow-up. Although modest and not statistically significant, the observed increase is likely to have beneficial health consequences. $\mathrm{VO}_{2} \max$ is a cardinal determinant of insulin sensitivity of skeletal muscle and in subcutaneous adipose tissue. ${ }^{1}$ Therefore, increasing $\mathrm{VO}_{2} \max$ in this population may result in increased insulin sensitivity in the tissues and thus improved glycaemic control. In addition, increasing aerobic power in people with T2D is related to a less atherogenic lipid profile, which may lessen the accelerated rate of atherosclerosis and other cardiovascular mortalities in this population. ${ }^{1}$ Increases in peak isometric quadriceps femoris torque were also demonstrated following the intervention. Combined training protocols in $\mathrm{T} 2 \mathrm{D}$ have shown strength increases that are comparable to those found in this study. ${ }^{21}$ The modest increases in both strength (14\%) and $\mathrm{VO}_{2} \max (3 \%)$ described in this study reflect the intensity of NMES training. Higher intensity training, which is exclusively resistance or aerobic in nature, may result in greater improvements in strength and $\mathrm{VO}_{2} \mathrm{max}$, respectively.

In this study, blood pressure, resting heart rate and lipid profile did not significantly improve following the NMES exercise intervention. This is consistent with another study which investigated the effect of an 8-week combined aerobic and resistance training programme and found no significant differences in plasma lipids and blood pressure. ${ }^{21}$ The duration of the training intervention may not have been sufficient to exact changes in these variables. Also, a potential confounder may be the relatively good baseline blood pressure and lipid profile of those who participated.

Previous reports investigating the potential use of NMES exercise in this population have found limitations in its use due its poor tolerability. ${ }^{13}$ The stimulation used in this investigation was, however, generally well tolerated with a good level of compliance ( $\geq 83 \%)$. Compliance levels were, however, self-reported and therefore participants may have overstated the actual number of training sessions they undertook and the intensity of training. 
Despite the positive findings of this investigation, there are a number of limitations to this study and some considerations for future studies which must be highlighted:

- This study was a single-group cohort intervention study with no control group for comparison. Comparing the NMES intervention group with a 'resting' control group would have allowed for more definitive conclusions about the effects of this intervention to be drawn. In addition, to determine whether NMES exercise is a suitable alternative to conventional exercise, a comparison between NMES exercise and conventional exercise should have been undertaken. In addition, to allow for comparisons with conventional exercise, future studies should examine the metabolic adaptations to NMES exercise - for example, the change in concentration of exercise regulated myokines before and after NMES exercise should be investigated.

- Diet and physical activity were not controlled for during the 8-week intervention period, therefore their positive effects cannot be excluded in the current study.

- The improvements in fasting plasma glucose levels observed in this study were from blood samples taken before and after the 8-week intervention period. It is possible that the observed change in fasting plasma glucose levels was due to an acute response to the NMES intervention rather than an accumulative training effect over the 8-week intervention period. Future NMES intervention studies should employ more frequent blood sampling in order to examine this.

- While the NMES intervention used in this study was shown to reduce body fat percentage, this study did not examine whether the lowering of total percentage body fat also resulted in improved plasma NEFA levels. Future studies examining the response to NMES in T2D patients should assess NEFA before and after the intervention.

- The study only included a small number of participants and was limited to non-insulin requiring T2D patients. Participants were relatively healthy and were not severely affected by cardiovascular disease or other diabetic complications. This may explain why some of the changes observed were modest and not statistically significant.

- Only male participants were recruited as previous pilot testing demonstrated that this intervention was not well tolerated by overweight/obese female patients with $T 2 D$, therefore these results cannot be generalised to the entire T2D population.

- Improvements in glucose sensitivity and/or pancreatic betacell function are the ultimate goal in the treatment, management and reversibility of T2D. Future studies examining the response to NMES in this population should explore these parameters. In addition, the possible mechanism of NMES exercise-induced improvements in glycaemic control is currently unknown. Future NMES intervention studies with T2D patients should also use glucose tolerance testing and/or hyperglycaemic and hyperinsulinaemic/euglycaemic clamping techniques to ascertain the possible mechanism of NMESinduced improvement in glycaemic control.

\section{Key messages}

- Neuromuscular electrical stimulation (NMES) may improve glycaemic control in type 2 diabetes

- NMES may improve body composition and strength in T2DM

- The findings are of particular relevance to those who have barriers to regular exercise or do not engage in the recommended amounts of regular exercise

\section{Conclusions}

Exercise is one of the main interventions known to help glycaemic control and prevent secondary complications in T2D. However, many individuals are unable or unwilling to exercise due to physical and mental barriers. This study suggests that NMES can be successfully used to elicit a physiological response in T2D consistent with traditional exercise. It therefore provides an attractive alternative to conventional exercise. However, a large randomised controlled trial is required to investigate this further.

Acknowledgement The authors would like to thank all those who participated in this study.

Contributors OMG performed the research and wrote the manuscript. LC developed the technology (with Bio-Medical Research Ltd) and parameters, and assisted with the acquisition of data. GFC contributed to the study design, data acquisition and assisted with the analysis of the data. BC supervised the study, contributing to the study design and the revision of the manuscript.

Conflict of interest This study was supported by Enterprise Ireland under the Innovation Partnership Programme and co-funded by Bio-Medical Research (BMR) Ltd. BMR Ltd supplied the NMES units used in this investigation.

Funding Work proceeding this study was supported and part-funded by Enterprise Ireland and Bio-Medical Research Ltd

Human and animal rights disclosure This study was approved by the Human Research Ethics Committees in University College Dublin and St Vincent's University Hospital, and was therefore performed in accordance with the ethical standards laid down in the 1964 Declaration of Helsinki and its later amendments.

Informed consent Informed consent was obtained from all patients included in the study.

\section{References}

1. Albright A, Franz M, Hornsby $G$, et al. Exercise and type 2 diabetes. Med Sci Sports Exercise 2000;32:1345-60. https://doi.org/10.1097/00005768-200007000-00024

2. America Diabetes Association. Standards of medical care in diabetes -2010 Diabetes Care 2010;33(Suppl 1):S11-61. https://doi.org/10.2337/dc10-S011

3. Sigal RJ, Kenny GP, Boule NG, et al. Effects of aerobic training, resistance training, or both on glycemic control in type 2 diabetes. Ann Intern Med 2007; 147:357-69.

https://doi.org/10.7326/0003-4819-147-6-200709180-00005

4. Thomas N, Adler E, Leese GP. Barriers to physical activity in patients with diabetes. Postgrad Med J 2004;80:287-91.

https://doi.org/10.1136/pgmj.2003.010553

5. Banerjee $P$, Caulfield B, Crowe L, Clark A. Prolonged electrical muscle stimulation exercise improves strength and aerobic capacity in healthy sedentary 
adults. J Appl Physio/ 2005;99:2307-11

https://doi.org/10.1152/japplphysiol.00891.2004

6. Banerjee P, Caulfield B, Crowe L, Clark A. Prolonged electrical muscle stimulation exercise improves strength, peak $\mathrm{VO}_{2}$ and exercise capacity in patients with stable chronic heart failure. J Card Fail 2009;15:319-26. http://dx.doi.org/10.1016/j.cardfail.2008.11.005

7. Grosset JF, Crowe L, De Vito G, et al. Comparative effect of a 1 h session of electrical muscle simulation and walking activity on energy expenditure and substrate oxidation in obese subjects. European College of Sport Science Congress, Oslo, 2009.

8. Hamada T, Sasaki H, Hayashi $T$, et al. Enhancement of whole body glucose uptake during and after human skeletal muscle low frequency electrical muscle stimulation. J Appl Physio/ 2003;94:2107-12.

https://doi.org/10.1152/japplphysiol.00486.2002

9. Hamada T, Hayashi T, Kimura T, et al. Electrical stimulation of human lower extremities enhances energy consumption, carbohydrate oxidation and whole body glucose uptake. J Appl Physiol 2004;96:9111-16.

10. Miyamoto T, Fukuda K, Kimura T, et al. Effect of percutaneous electrical muscle stimulation on postprandial hyperglycemia in type 2 diabetes. Diabetes Res Clin Pract 2012;96:306-12. http://dx.doi.org/10.1016/. diabres.2012.01.006

11. Jabbour G, Belliveau L, Probizanski $D$, et al. Effect of low frequency neuromuscular electrical stimulation on glucose profile of persons with type 2 diabetes: a pilot study. Diabetes Metab J 2015;39:264-7. http://dx.doi.org/10.4093/dmj.2015.39.3.264

12. Joubert $M$, Metayer $L$, Prevost $G$, et al. Neuromuscular electrostimulation and insulin sensitivity in patients with type 2 diabetes: the ELECTODIAB pilot study. Acta Diabetol 2015;52:285-91.

http://dx.doi.org/10.1007/s00592-014-0636-5

13. Poole RB, Harold CP, Burridge JH, Byrne CD, Holt RI. Electrical muscle stimulation acutely mimics exercise in neurologically intact individuals but has limited clinical benefits in patients with type 2 diabetes. Diabetes Obes
Metab 2005;7:344-51. https://doi.org/10.1111/j.1463-1326.2004.00400.x

14. Doheny E, Caulfield B, Minogue C, Lowery M. The effect of subcutaneous fat thickness on the efficacy of transcutaneous electrical stimulation. 30th Annual International IEEE EMBS Conference, 2008. https://doi.org/10.1109/iembs.2008.4650504

15. Wasserman K, Hansen JE, Sue DY, et al. Principles of exercise testing and interpretation: including pathophysiology and clinical applications. Volume 206. Philadelphia: Lippincott Williams \& Wilkins, 1999.

16. Ohkubo $Y$, Kishikawa $H$, Araki $E$, et al. Intensive insulin therapy prevents the progression of diabetes microvascular complications in Japanese patients with non-insulin-dependent diabetes mellitus: a randomized prospective 6year study. Diabetes Res Clin Pract 1995;28:103-17. https://doi.org/10.1016/0168-8227(95)01064-K

17. Crowe L, Caulfield B. Aerobic neuromuscular electrical stimulation - an emerging technology to improve haemoglobin $A 1 \mathrm{C}$ in type 2 diabetes mellitus: results of a pilot study. BMJ Open 2012;2(3):e000219. http://dx.doi.org/10.1136/bmjopen-2011-000219

18. Church TS, Blair SN, Cocreham S, et al. Effects of aerobic and resistance training on hemoglobin $\mathrm{A} 1 \mathrm{c}$ levels in patients with type 2 diabetes: a randomized controlled trial. JAMA 2010;304:2253-62. http://dx.doi.org/10.1001/jama.2010.1710

19. Yki-Jarvinen $H$. The insulin resistance syndrome. In: DeFronzo RA, Ferrannini $\mathrm{E}$, Keen $\mathrm{H}$, et al. International textbook of diabetes mellitus. 3rd edition. John Wiley \& Sons Ltd, 2004. https://doi.org/10.1002/ 0470862092.d0410

20. Santomauro AT, Boden G, Silva ME, et al. Overnight lowering of free fatty acids with Acipimox improves insulin resistance and glucose tolerance in obese diabetic and nondiabetic subjects. Diabetes 1999:48:1836-41. https://doi.org/10.2337/diabetes.48.9.1836

21. Maiorana A, O'Driscoll G, Goodman C, Taylor R, Green D. Combined aerobic and resistance exercise improves glycemic control and fitness in type 2 diabetes. Diabetes Res Clin Pract 2002;56:115-23. https://doi.org/10.1016/S0168-8227(01)00368-0

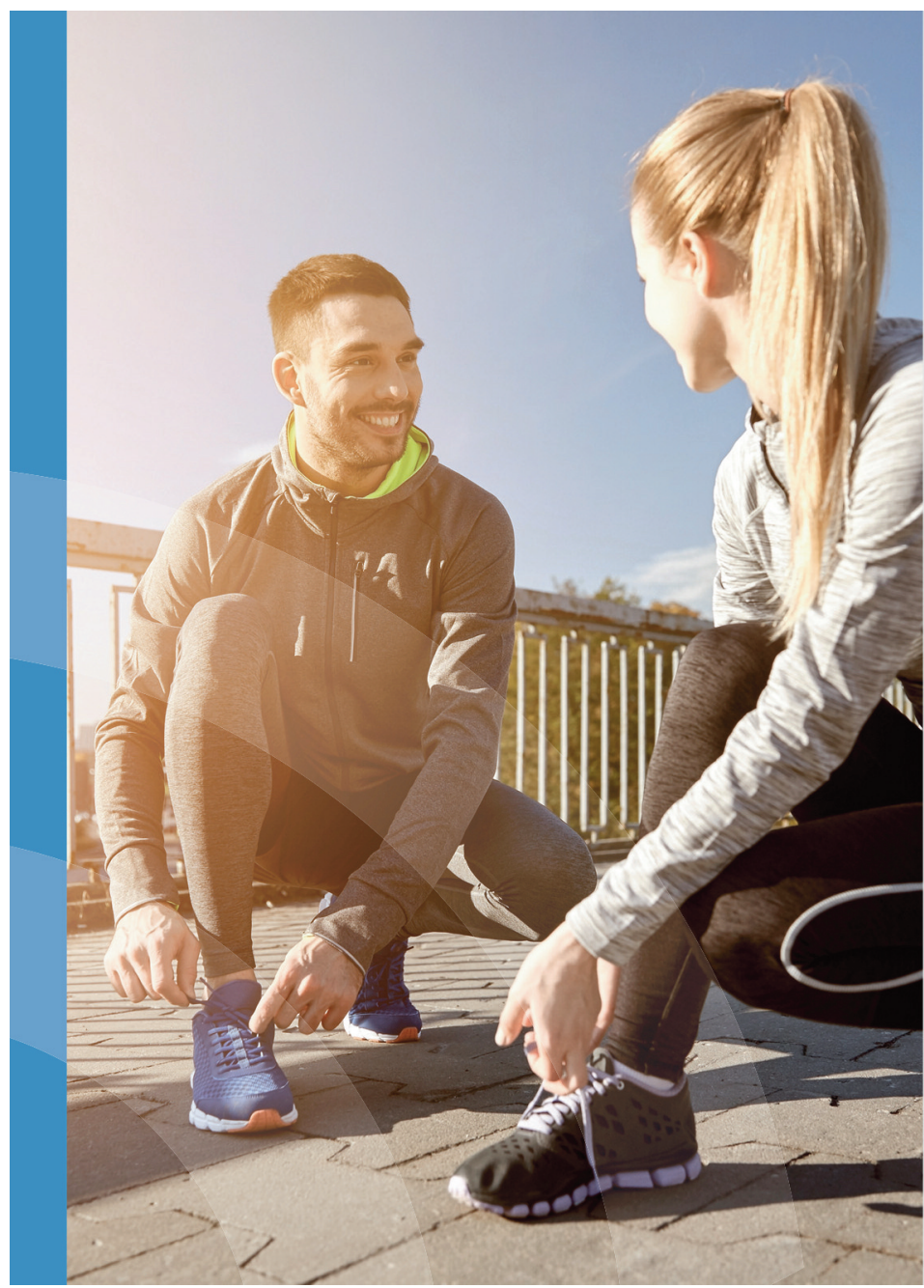

\section{cellñovo} There is afways a moment
you want to detach...

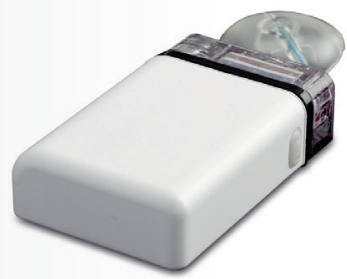

Diabetes is complicated enough.

Let's make it as simple as possible. 